\title{
General aspects of Hertzian potentials modeling in integrated optics
}

\author{
Alessandro Massaro ${ }^{1, *, \dagger}$ and Roberto Cingolani ${ }^{2}$ \\ ${ }^{1}$ Italian Institute of Technology IIT, Center of Bio-Molecular Nanotechnologies, Arnesano 73100, Italy \\ ${ }^{2}$ Italian Institute of Technology IIT, Via Morego 30, 16163 Genova, Italy
}

\begin{abstract}
SUMMARY
This work introduces a novel time-domain numerical simulator of integrated optical waveguides. Only solutions of two scalar Helmholtz-equations are used in the evaluation of electric and magnetic Hertzian-potentials that yields the electromagnetic field in the time domain, and the frequency response by the discrete Fourier transform. All the field components are obtained directly from the scalar potentials, and are not interrelated as in finite-difference time domain method. The Hertzian potential method (HPM) considers the field-perturbation effect in proximity of the dielectric discontinuities by generators modeling and takes into account the numerical error of a wave that travels in a dielectric multilayered optical guide. We present in this work the general finite difference discretization and the numerical setting of the HPM related to optical waveguide full-wave modeling. Copyright (C) 2011 John Wiley \& Sons, Ltd.
\end{abstract}

Received 29 December 2007; Revised 16 December 2010; Accepted 1 February 2011

KEY WORDS: Hertzian potentials; optical waveguide; design methodology; numerical and analytical approximation

\section{INTRODUCTION}

Time domain methods such as finite-difference time-domain (FDTD) [1, 2], transmission line matrix [3], and wavelet-Galerkin method [4] are gaining importance by virtue of their versatility and represent the natural way in which they simulate what happens in electromagnetic (EM) problems. For high-frequency optical structures, the complexity of problems grows, and a high computer performance is requested. The rigorous Hertzian Potentials formulation can be used for simulation of full-wave propagation and reflection in the time domain, it is not time- and memory-intensive and is suitable for structure of large optical dimensions [5, 6]. We propose an efficient numerical algorithm to solve the EM field in a general optical waveguide by considering the typical analysis of the optical waveguides. This method considers the Hertz vectors [7-9] starting from the Helmholtz scalar equations $[9,10]$ and the perturbed effect of the dielectric discontinuities [11-13]. The equations to solve are reduced to two scalar equations instead of six; subsequently all the EM components are obtained by the Hertzian potentials [7-9]. In the frequency domain, the transmission and reflection properties of dielectric discontinuities may be derived by means of an equivalent circuit [11-13] that automatically ensures continuity of the fields and their first derivatives along the propagation-directions. If potentials are used,

\footnotetext{
*Correspondence to: Alessandro Massaro, Italian Institute of Technology IIT, Center of Bio-Molecular Nanotechnologies, Arnesano 73100, Italy.

${ }^{\dagger}$ E-mail: alessandro.massaro@iit.it
} 
instead, second derivatives are involved and generators are necessary at each dielectric interface. The generators $[5,6]$ decrease the grid cell dimension and so the computational time with a good convergent solution [6]. The method is developed by considering the modal approach of the waveguides shown in Figure 1, by evaluating the effective refractive index along the nonuniform dielectric direction, and then by modeling the waveguides by the Hertzian potential formulation. The analytical model (used to reduce a 3D problem in $2 \mathrm{D}$ one through the effective index of the vertical direction), and the numerical time-domain Hertzian Potentials Method (HPM) model with generators represent together a good approach for simulating complex 3D structures that require a high computational cost [6]. In this work, we describe in detail the application of the Hertzian potentials in a general optical waveguide by focusing the theory in the evaluation of important parameters related to the waveguide analysis.

\section{TIME DOMAIN HERTZIAN POTENTIALS METHOD (HPM)}

In Figure 2 the time-domain Hertzian Potentials algorithm used in this work [5, 6] is shown. The Hertzian electric and magnetic vectors [7-9] are represented in rectangular coordinates by

$$
\begin{aligned}
& \overline{\Pi_{\mathrm{e}}}=\mathbf{a} \psi_{\mathrm{e}}(x, y, z, t) \\
& \overline{\Pi_{\mathrm{h}}}=\mathbf{a} \psi_{\mathrm{h}}(x, y, z, t)
\end{aligned}
$$

where $\mathbf{a}$ is unit vector. From (1) it is possible to evaluate all the components of the EM field as

$$
\begin{aligned}
& \overline{\mathbf{E}}=\nabla \nabla \cdot \bar{\Pi}_{\mathrm{e}}-\varepsilon \mu \frac{\partial^{2}}{\partial t^{2}} \bar{\Pi}_{\mathrm{e}}-\mu \frac{\partial}{\partial t}\left(\nabla \times \bar{\Pi}_{\mathrm{h}}\right) \\
& \overline{\mathbf{H}}=\nabla \nabla \cdot \bar{\Pi}_{\mathrm{h}}-\varepsilon \mu \frac{\partial^{2}}{\partial t^{2}} \bar{\Pi}_{\mathrm{h}}+\varepsilon \frac{\partial}{\partial t}\left(\nabla \times \bar{\Pi}_{\mathrm{e}}\right)
\end{aligned}
$$

where

$$
\begin{aligned}
\nabla \cdot \bar{\Pi}_{\mathrm{e}, \mathrm{h}} & =\partial_{x} \psi^{\mathrm{e}, \mathrm{h}}+\partial_{y} \psi^{\mathrm{e}, \mathrm{h}}+\partial_{z} \psi^{\mathrm{e}, \mathrm{h}} \\
\nabla \nabla \cdot \bar{\Pi}_{\mathrm{e}, \mathrm{h}} & =\hat{x} A^{\mathrm{e}, \mathrm{h}}+\hat{y} B^{\mathrm{e}, \mathrm{h}}+\hat{z} C^{\mathrm{e}, \mathrm{h}} \\
\nabla \times \bar{\Pi}_{\mathrm{e}, \mathrm{h}} & =\hat{x}\left(\partial_{y} \psi^{\mathrm{e}, \mathrm{h}}-\partial_{z} \psi^{\mathrm{e}, \mathrm{h}}\right)+\hat{y}\left(\partial_{z} \psi^{\mathrm{e}, \mathrm{h}}-\partial_{x} \psi^{\mathrm{e}, \mathrm{h}}\right)+\hat{z}\left(\partial_{x} \psi^{\mathrm{e}, \mathrm{h}}-\partial_{y} \psi^{\mathrm{e}, \mathrm{h}}\right)
\end{aligned}
$$

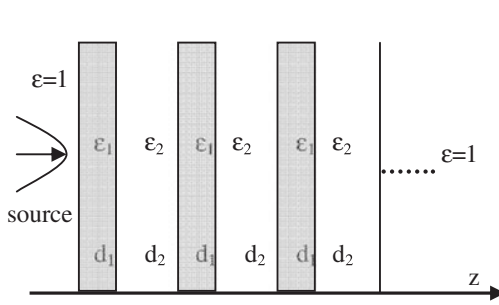

(a)

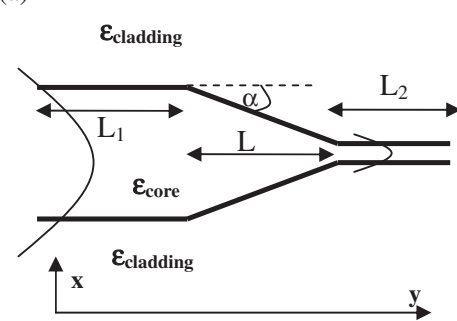

(c)

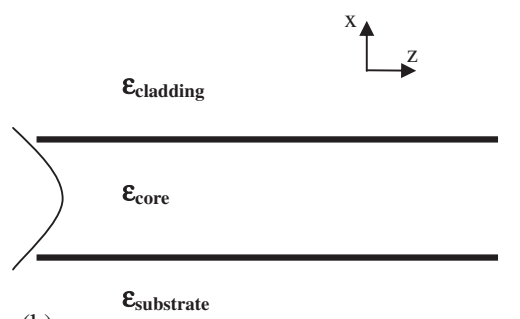

(b)

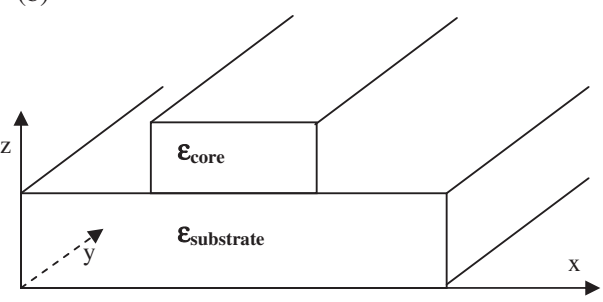

(d)

Figure 1. (a) 1D dielectric multilayer structure; (b) 2D asymmetrical waveguide; (c) 2D tapered waveguide; and (d) 3D ridge waveguide. 


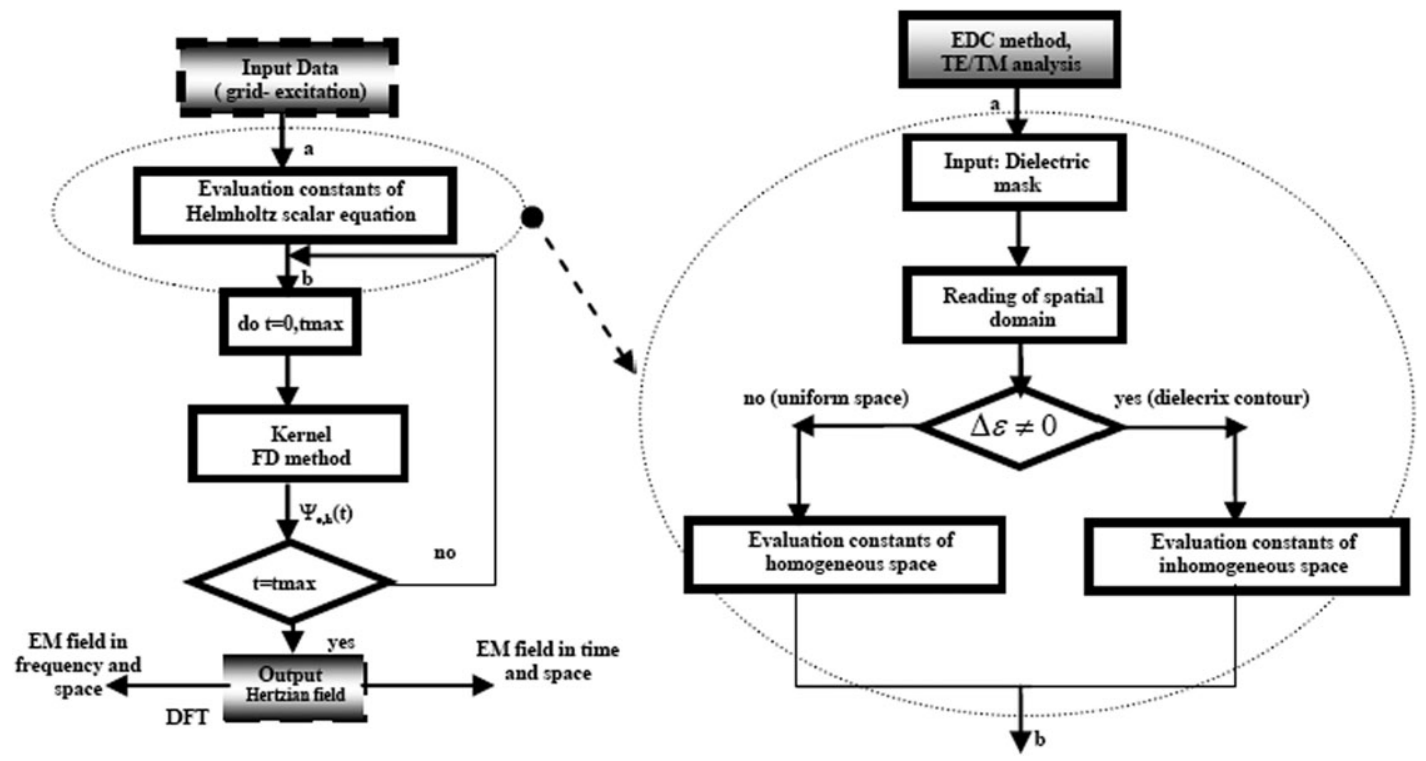

Figure 2. 2D HPM algorithm with analytical approximation: the input parameters (thicknesses, refractive indices, working wavelength) are given after the analysis of the symmetrical and the asymmetrical slab waveguide.

with

$$
\begin{aligned}
& A^{\mathrm{e}, \mathrm{h}}=\partial_{x}^{2} \psi^{\mathrm{e}, \mathrm{h}}+\partial_{y x} \psi^{\mathrm{e}, \mathrm{h}}+\partial_{z x} \psi^{\mathrm{e}, \mathrm{h}} \\
& B^{\mathrm{e}, \mathrm{h}}=\partial_{x y} \psi^{\mathrm{e}, \mathrm{h}}+\partial_{y}^{2} \psi^{\mathrm{e}, \mathrm{h}}+\partial_{z y} \psi^{\mathrm{e}, \mathrm{h}} \\
& C^{\mathrm{e}, \mathrm{h}}=\partial_{x z} \psi^{\mathrm{e}, \mathrm{h}}+\partial_{y z} \psi^{\mathrm{e}, \mathrm{h}}+\partial_{z}^{2} \psi^{\mathrm{e}, \mathrm{h}}
\end{aligned}
$$

By using (3) and (4), Equation (2) becomes

$$
\begin{aligned}
\overline{\mathbf{E}}= & \hat{x} A^{\mathrm{e}}+\hat{y} B^{\mathrm{e}}+\hat{z} C^{\mathrm{e}}-\hat{x} \mu \varepsilon \partial_{t}^{2} \psi^{\mathrm{e}}-\hat{y} \mu \varepsilon \partial_{t}^{2} \psi^{\mathrm{e}}-\hat{z} \mu \varepsilon \partial_{t}^{2} \psi^{\mathrm{e}} \\
& -\mu \partial_{t}\left(\hat{x}\left(\partial_{y} \psi^{\mathrm{h}}-\partial_{z} \psi^{\mathrm{h}}\right)+\hat{y}\left(\partial_{z} \psi^{\mathrm{h}}-\partial_{x} \psi^{\mathrm{h}}\right)+\hat{z}\left(\partial_{x} \psi^{\mathrm{h}}-\partial_{y} \psi^{\mathrm{h}}\right)\right) \\
\overline{\mathbf{H}}= & \hat{x} A^{\mathrm{h}}+\hat{y} B^{\mathrm{h}}+\hat{z} C^{\mathrm{h}}-\hat{x} \mu \varepsilon \partial_{t}^{2} \psi^{\mathrm{h}}-\hat{y} \mu \varepsilon \partial_{t}^{2} \psi^{\mathrm{h}}-\hat{z} \mu \varepsilon \partial_{t}^{2} \psi^{\mathrm{h}} \\
& +\varepsilon \partial_{t}\left(\hat{x}\left(\partial_{y} \psi^{\mathrm{e}}-\partial_{z} \psi^{\mathrm{e}}\right)+\hat{y}\left(\partial_{z} \psi^{\mathrm{e}}-\partial_{x} \psi^{\mathrm{e}}\right)+\hat{z}\left(\partial_{x} \psi^{\mathrm{e}}-\partial_{y} \psi^{\mathrm{e}}\right)\right)
\end{aligned}
$$

All the EM components of Equation (5) for a general unit vector a are given by:

$$
\begin{aligned}
E_{x} & =A^{\mathrm{e}}-\mu \varepsilon \partial_{t}^{2} \psi^{\mathrm{e}}+\mu \partial_{t} \partial_{z} \psi^{\mathrm{h}}-\mu \partial_{t} \partial_{y} \psi^{\mathrm{h}} \\
E_{y} & =B^{\mathrm{e}}-\mu \varepsilon \partial_{t}^{2} \psi^{\mathrm{e}}-\mu \partial_{t} \partial_{z} \psi^{\mathrm{h}}+\mu \partial_{t} \partial_{x} \psi^{\mathrm{h}} \\
E_{z} & =C^{\mathrm{e}}-\mu \varepsilon \partial_{t}^{2} \psi^{\mathrm{e}}-\mu \partial_{t} \partial_{x} \psi^{\mathrm{h}}+\mu \partial_{t} \partial_{y} \psi^{\mathrm{h}} \\
H_{x} & =A^{\mathrm{h}}-\mu \varepsilon \partial_{t}^{2} \psi^{\mathrm{h}}+\varepsilon \partial_{t} \partial_{y} \psi^{\mathrm{e}}-\varepsilon \partial_{t} \partial_{z} \psi^{\mathrm{e}} \\
H_{y} & =B^{\mathrm{h}}-\varepsilon \mu \partial_{t}^{2} \psi^{\mathrm{h}}+\varepsilon \partial_{t} \partial_{z} \psi^{\mathrm{e}}-\varepsilon \partial_{t} \partial_{x} \psi^{\mathrm{e}} \\
H_{z} & =C^{\mathrm{h}}-\mu \varepsilon \partial_{t}^{2} \psi^{\mathrm{h}}+\varepsilon \partial_{t} \partial_{x} \psi^{\mathrm{e}}-\varepsilon \partial_{t} \partial_{y} \psi^{\mathrm{e}}
\end{aligned}
$$

The potentials $\Psi^{\mathrm{e}, \mathrm{h}}(x, y, z, t)$ represent the solutions of the homogeneous wave equations for a non-dissipative medium [5]

$$
\nabla^{2} \psi_{\mathrm{e}, \mathrm{h}}(x, y, z, t)-\mu \varepsilon \frac{\partial^{2} \psi_{\mathrm{e}, \mathrm{h}}(x, y, z, t)}{\partial t^{2}}=0
$$

and for a dissipative medium

$$
\nabla^{2} \psi_{\mathrm{e}, \mathrm{h}}(x, y, z, t)-\mu \varepsilon \frac{\partial^{2} \psi_{\mathrm{e}, \mathrm{h}}(x, y, z, t)}{\partial t^{2}}-\mu \sigma \frac{\partial \psi_{\mathrm{e}, \mathrm{h}}(x, y, z, t)}{\partial t}=0
$$


where $\varepsilon$ is the electrical permittivity $(\mathrm{F} / \mathrm{m}), \mu$ is magnetic permeability $(\mathrm{H} / \mathrm{m})$, and $\sigma$ is the electric conductivity $(\mathrm{S} / \mathrm{m})$ which is zero in a perfect dielectric.

For the solution of Equations (7) and (8), we use the finite difference (FD) discretization [14], which for a general three-dimensional $(x, y)$ space is represented by

$$
\begin{aligned}
& \frac{\partial \psi_{\mathrm{e}, \mathrm{h}}^{n}(i, j, k)}{\partial x}=\frac{\psi_{\mathrm{e}, \mathrm{h}}^{n}(i+1 / 2, j, k)-\psi_{\mathrm{e}, \mathrm{h}}^{n}(i-1 / 2, j, k)}{\Delta x}+O\left(\Delta^{2} x\right) \\
& \frac{\partial \psi_{\mathrm{e}, \mathrm{h}}^{n}(i, j, k)}{\partial y}=\frac{\psi_{\mathrm{e}, \mathrm{h}}^{n}(i, j+1 / 2, k)-\psi_{\mathrm{e}, \mathrm{h}}^{n}(i, j-1 / 2, k)}{\Delta y}+O\left(\Delta^{2} y\right) \\
& \frac{\partial \psi_{\mathrm{e}, \mathrm{h}}^{n}(i, j, k)}{\partial z}=\frac{\psi_{\mathrm{e}, \mathrm{h}}^{n}(i, j, k+1 / 2)-\psi_{\mathrm{e}, \mathrm{h}}^{n}(i, j, k-1 / 2)}{\Delta y}+O\left(\Delta^{2} z\right) \\
& \frac{\partial \psi_{\mathrm{e}, \mathrm{h}}^{n}(i, j, k)}{\partial t}=\frac{\psi_{\mathrm{e}, \mathrm{h}}^{n+1 / 2}(i, j, k)-\psi_{\mathrm{e}, \mathrm{h}}^{n-1 / 2}(i, j, k)}{\Delta t}+O\left(\Delta^{2} t\right)
\end{aligned}
$$

and for the second order by:

$$
\begin{aligned}
& \frac{\partial^{2} \psi_{\mathrm{e}, \mathrm{h}}^{n}(i, j, k)}{\partial x^{2}} \simeq \frac{\psi_{\mathrm{e}, \mathrm{h}}^{n}(i+1, j, k)-2 \psi_{\mathrm{e}, \mathrm{h}}^{n}(i, j, k)+\psi_{\mathrm{e}, \mathrm{h}}^{n}(i-1, j, k)}{\Delta^{2} x} \\
& \frac{\partial^{2} \psi_{\mathrm{e}, \mathrm{h}}^{n}(i, j, k)}{\partial y^{2}} \simeq \frac{\psi_{\mathrm{e}, \mathrm{h}}^{n}(i, j+1, k)-2 \psi_{\mathrm{e}, \mathrm{h}}^{n}(i, j, k)+\psi_{\mathrm{e}, \mathrm{h}}^{n}(i, j-1, k)}{\Delta^{2} y} \\
& \frac{\partial^{2} \psi_{\mathrm{e}, \mathrm{h}}^{n}(i, j, k)}{\partial z^{2}} \simeq \frac{\psi_{\mathrm{e}, \mathrm{h}}^{n}(i, j, k+1)-2 \psi_{\mathrm{e}, \mathrm{h}}^{n}(i, j, k)+\psi_{\mathrm{e}, \mathrm{h}}^{n}(i, j, k-1)}{\Delta^{2} z} \\
& \frac{\partial^{2} \psi_{\mathrm{e}, \mathrm{h}}^{n}(i, j, k)}{\partial t^{2}} \simeq \frac{\psi_{\mathrm{e}, \mathrm{h}}^{n+1}(i, j, k)-2 \psi_{\mathrm{e}, \mathrm{h}}^{n}(i, j, k)+\psi_{\mathrm{e}, \mathrm{h}}^{n-1}(i, j, k)}{\Delta^{2} t}
\end{aligned}
$$

\section{HPM GENERATORS MODELING FOR DIELECTRIC DISCONTINUITIES}

It is known that the scalar wave equation may lead to inconsistencies because in an inhomogeneous medium, it is, in general, not equivalent to Maxwell's equations. EM scattering problems, including free space, involve the calculation of the fields produced in the presence of geometrical discontinuities by arbitrary currents and voltages (generators). Such discontinuities may be replaced by equivalent generators [4], giving an accurate solution of the EM fields for structures with high dielectric contrast. In fact the scalar wave equation (7) for a non-dissipative medium can be rewritten as [5]

$$
\nabla^{2} \Psi_{\mathrm{e}, \mathrm{h}}(x, y, z, t)-\mu \varepsilon \frac{\partial^{2} \Psi_{\mathrm{e}, \mathrm{h}}(x, y, z, t)}{\partial t^{2}}-\mu \frac{\partial^{2} P_{\mathrm{pert}}(x, y, z, t)}{\partial t^{2}}=0
$$

where

$$
P_{\text {pert }}(x, y, z, t)=\Delta \varepsilon(x, y, z, t) \Psi_{\mathrm{e}, \mathrm{h}}(x, y, z, t)
$$

represents the dielectric polarization, and for a $3 \mathrm{D}$ case we have:

$$
\begin{aligned}
& \Delta \varepsilon=\varepsilon_{i+1}-\varepsilon_{i}, \quad i=\text { cell position in the } x-\text { direction } \\
& \Delta \varepsilon=\varepsilon_{j+1}-\varepsilon_{j}, \quad j=\text { cell position in the } y \text {-direction } \\
& \Delta \varepsilon=\varepsilon_{k+1}-\varepsilon_{k}, \quad k=\text { cell position in the } z \text { - direction }
\end{aligned}
$$

Therefore, we solve (11) in proximity of the dielectric interfaces, and (7) in the homogenous regions. The difference between the parametric solution of (11), and (7) in the iterative form (by using the FD discretization [14]), is in the coefficients [5, 6]; in fact for one propagation direction, (7) and (11) become, respectively, 


$$
\begin{aligned}
\psi^{n+1}(j)\left(\frac{\mu \varepsilon}{(\Delta t)^{2}}\right)= & \psi^{n}(j+1)\left(\frac{1}{(\Delta z)^{2}}\right)+\psi^{n}(j)\left(\frac{2 \mu \varepsilon}{(\Delta t)^{2}}-\frac{2}{(\Delta z)^{2}}\right) \\
& +\psi^{n-1}(j)\left(-\frac{\mu \varepsilon}{(\Delta t)^{2}}\right)+\psi^{n}(j-1)\left(\frac{1}{(\Delta z)^{2}}\right) \\
\psi^{n+1}(j)\left(\frac{\mu \varepsilon}{(\Delta t)^{2}}+\frac{\mu \Delta \varepsilon}{(\Delta t)^{2}}\right)= & \psi^{n}(j+1)\left(\frac{1}{(\Delta z)^{2}}\right)+\psi^{n}(j)\left(\frac{2 \mu \varepsilon}{(\Delta t)^{2}}-\frac{2}{(\Delta z)^{2}}+\frac{2 \mu \Delta \varepsilon}{(\Delta t)^{2}}\right) \\
& +\psi^{n-1}(j)\left(-\frac{\mu \varepsilon}{(\Delta t)^{2}}-\frac{\mu \Delta \varepsilon}{(\Delta t)^{2}}\right)+\psi^{n}(j-1)\left(\frac{1}{(\Delta z)^{2}}\right)
\end{aligned}
$$

and by considering the implemented formula

$$
\begin{aligned}
& \psi^{n+1}(j)=\psi^{n}(j+1)\left(\frac{b}{a}\right)+\psi^{n}(j)\left(\frac{2 a-2 b}{a}\right)+\psi^{n-1}(j)(-1)+\psi^{n}(j-1)\left(\frac{b}{a}\right) \\
& \psi^{n+1}(j)=\psi^{n}(j+1)\left(\frac{b}{a^{\prime}}\right)+\psi^{n}(j)\left(\frac{2 a^{\prime}-2 b}{a^{\prime}}\right)+\psi^{n-1}(j)(-1)+\psi^{n}(j-1)\left(\frac{b}{a^{\prime}}\right)
\end{aligned}
$$

with

$$
\begin{aligned}
a & =\frac{\mu \varepsilon}{(\Delta t)^{2}} \\
a^{\prime} & =a+\frac{\mu \Delta \varepsilon}{(\Delta t)^{2}} \\
b & =\frac{1}{(\Delta z)^{2}}
\end{aligned}
$$

Figure 3 shows the time evolution of the $E_{y}$ component (normal to plane of the figure) one cell before the dielectric multilayer 1D of Figure 1(a). We observe that the parameters (20) represent the values of the generators (see inset of Figure 3) and give a good convergent solution with a low grid size [6], in fact, it is clear how by using a no-small cell size $\left(\Delta z=4 \times 10^{-8} \mathrm{~m}\right)$ with respect to the dielectric thicknesses the generators provide a no-oscillating closed solution.

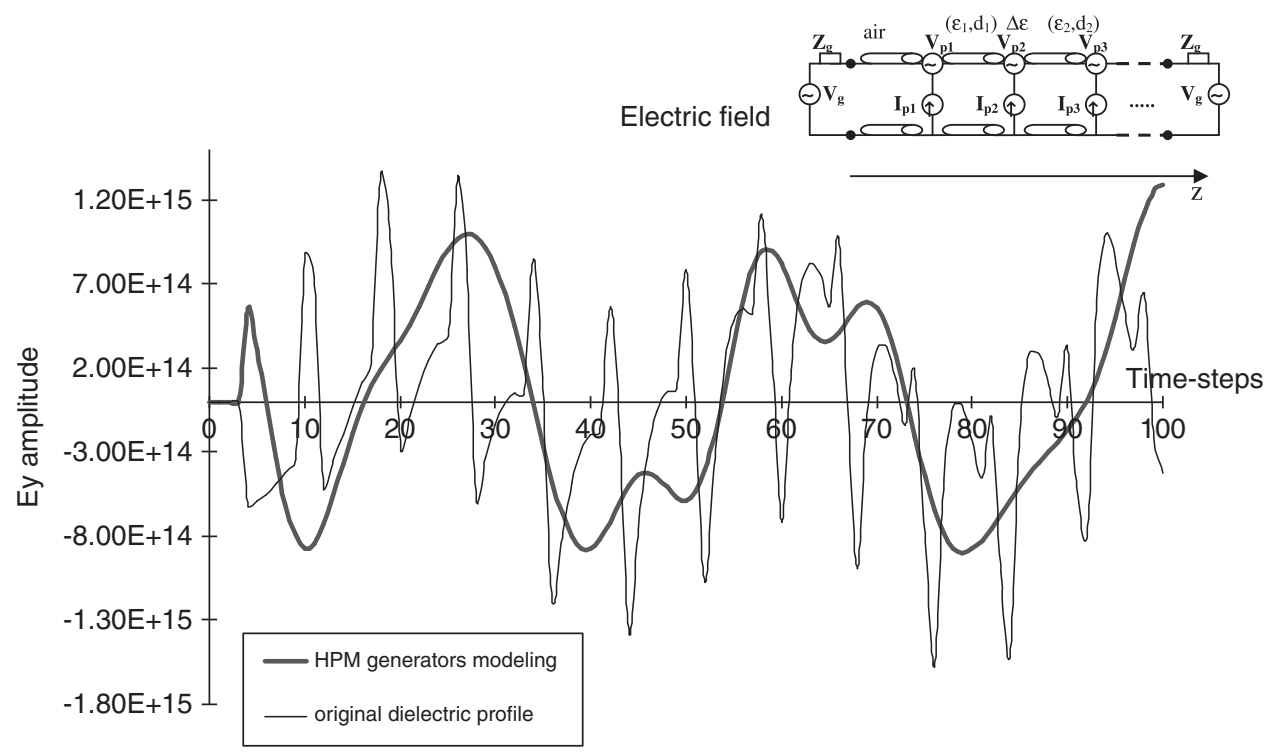

Figure 3. Time evolution of $E_{y}$ field component one cell before the dielectric multilayer structure of Figure 1(a). In particular the multilayer structure is characterized by 15-layer of $\mathrm{Si}$ and $\mathrm{SiO}_{2}$ materials, $n_{1}=3.48$, $d_{1}=0.11 \mu \mathrm{m}, n_{2}=1.44, d_{2}=0.26 \mu \mathrm{m}$. The structure is discretized by 70 domain cells with $\Delta z=4 \times 10^{-8} \mathrm{~m}$, $\mathrm{d} t=1.33 \times 10^{-16} \mathrm{~s}$, the first five cells and the last five ones are filled by air. The input signal is a sinusoidal signal with working wavelength $1.523 \mu \mathrm{m}$ modulated by a Gaussian pulse. 
The oscillations, which represent numerical errors, will not appear with a very fine space discretization but this requires a high computational cost [6].

\section{ABSORBING BOUNDARY CONDITIONS AND GENERAL SETTING}

The absorbing boundary conditions (ABCs) [14, 15], around the computational domain $\Omega=(x, y, z)$, permit all outward-propagating numerical waves to exit $\Omega$ as if the simulation were performed on a computational domain of infinite extent. In the HPM kernel, we use for the implementation of (8), (9), and (12) in a matrix structure as

$\downarrow \rightarrow i, j$ space domain position

$$
\left(\begin{array}{ccccccccc}
\mathrm{ABC} & \mathrm{ABC} & \mathrm{ABC} & \mathrm{ABC} & \mathrm{ABC} & \mathrm{ABC} & \mathrm{ABC} & \mathrm{ABC} & \mathrm{ABC} \\
\mathrm{ABC} & \cdot & \cdot & \psi^{n}(i, j) & \psi^{n}(i+1, j) & \psi^{n}(i+2, j) & \cdot & \cdot & \mathrm{ABC} \\
\mathrm{ABC} & \cdot & \cdot & \cdot & \psi^{n}(i+1, j+1) & \cdot & \cdot & \psi^{n}(m, j+1) & \mathrm{ABC} \\
\mathrm{ABC} & \cdot & \cdot & \cdot & \cdot & \cdot & \cdot & \cdot & \mathrm{ABC} \\
\mathrm{ABC} & \cdot & \cdot & \cdot & \psi^{n}(i+1, l) & \cdot & \cdot & \cdot & \mathrm{ABC} \\
\mathrm{ABC} & \mathrm{ABC} & \mathrm{ABC} & \mathrm{ABC} & \mathrm{ABC} & \mathrm{ABC} & \mathrm{ABC} & \mathrm{ABC} & \mathrm{ABC}
\end{array}\right)^{m \times l}
$$

where $m \times l$ is the nodes number of the spatial domain, time-step and the $k$ coordinate are fixed, and $\mathrm{ABC}$ indicates the $\mathrm{ABCs}$ Mur boundary conditions $[14,15]$. ABCs consider the wave equation:

$$
\begin{array}{ll}
\frac{\partial^{2} \psi_{\mathrm{e}, \mathrm{h}}}{\partial x \partial t}-\frac{1}{c} \frac{\partial^{2} \psi_{\mathrm{e}, \mathrm{h}}}{\partial t^{2}}+\frac{c}{2} \frac{\partial^{2} \psi_{\mathrm{e}, \mathrm{h}}}{\partial y^{2}}=0, & x=0 \\
\frac{\partial^{2} \psi_{\mathrm{e}, \mathrm{h}}}{\partial x \partial t}+\frac{1}{c} \frac{\partial^{2} \psi_{\mathrm{e}, \mathrm{h}}}{\partial t^{2}}+\frac{c}{2} \frac{\partial^{2} \psi_{\mathrm{e}, \mathrm{h}}}{\partial y^{2}}=0, & x=n_{x} \\
\frac{\partial^{2} \psi_{\mathrm{e}, \mathrm{h}}}{\partial y \partial t}-\frac{1}{c} \frac{\partial^{2} \psi_{\mathrm{e}, \mathrm{h}}}{\partial t^{2}}+\frac{c}{2} \frac{\partial^{2} \psi_{\mathrm{e}, \mathrm{h}}}{\partial x^{2}}=0, & y=0 \\
\frac{\partial^{2} \psi_{\mathrm{e}, \mathrm{h}}}{\partial y \partial t}+\frac{1}{c} \frac{\partial^{2} \psi_{\mathrm{e}, \mathrm{h}}}{\partial t^{2}}-\frac{c}{2} \frac{\partial^{2} \psi_{\mathrm{e}, \mathrm{h}}}{\partial x^{2}}=0, & y=n_{y} \\
\frac{\partial^{2} \psi_{\mathrm{e}, \mathrm{h}}}{\partial z \partial t}-\frac{1}{c} \frac{\partial^{2} \psi_{\mathrm{e}, \mathrm{h}}}{\partial t^{2}}+\frac{c}{2} \frac{\partial^{2} \psi_{\mathrm{e}, \mathrm{h}}}{\partial z^{2}}=0, & z=0 \\
\frac{\partial^{2} \psi_{\mathrm{e}, \mathrm{h}}}{\partial z \partial t}+\frac{1}{c} \frac{\partial^{2} \psi_{\mathrm{e}, \mathrm{h}}}{\partial t^{2}}+\frac{c}{2} \frac{\partial^{2} \psi_{\mathrm{e}, \mathrm{h}}}{\partial z^{2}}=0, & z=n_{z}
\end{array}
$$

where $x=0, n_{x}, y=0, n_{y}, z=0, n_{z}$ represent the boundary-coordinates of a three-dimensional domain $(x, y, z)$. To obtain a stable simulation, we consider a temporal step size condition $[14,15]$

$$
\mathrm{cdt}<\frac{1}{\sqrt{\frac{1}{\Delta x^{2}}+\frac{1}{\Delta y^{2}}+\frac{1}{\Delta z^{2}}}}
$$

A key part of an HPM simulation is the launch field used to excite the structure. The excitation, also referred to as the launched field, should be chosen to correspond to the type of simulation results required. For example, a continuous wave excitation:

$$
\Psi_{\text {source }}^{\mathrm{e}, \mathrm{h}}=\sin (\omega \cdot t \cdot \mathrm{d} t)
$$

should be used when the steady-state performance of a structure is desired, and a pulsed excitation (carrier modulated by an exponential field)

$$
\Psi_{\text {source }}^{\mathrm{e}, \mathrm{h}}=\exp \left(-\left(t \cdot \mathrm{d} t / T_{0}\right)^{2}\right) \cdot \sin (\omega \cdot t \cdot \mathrm{d} t)
$$

can be used when the spectral characteristics of a structure are needed. In Figure 4 we show an example of Equation (25) in the time-domain evolution. We observe that in a $2 \mathrm{D}$ case the 


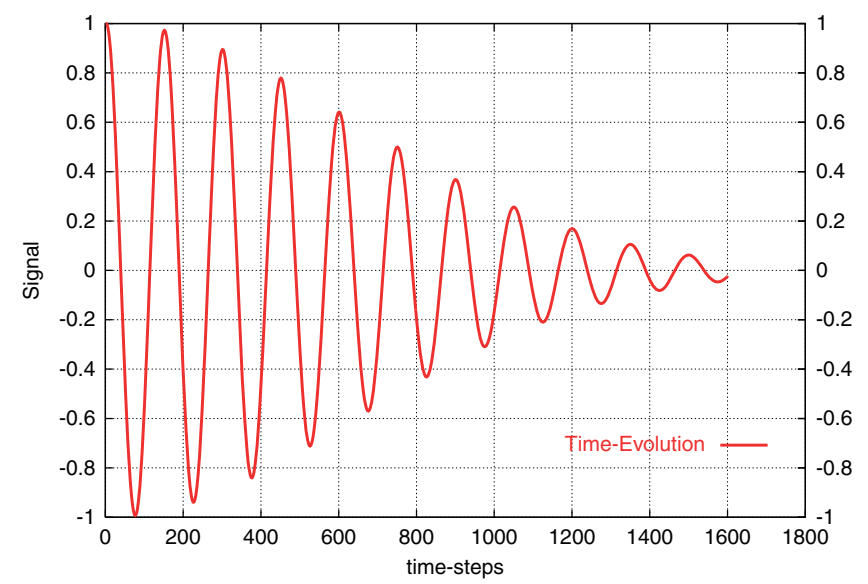

Figure 4. Example of input signal as sinusoidal signal with $\lambda_{0}=1.55 \mu \mathrm{m}$ and $T_{0}=30 \mathrm{ps}$, $\mathrm{d} t=1.18 \times 10^{-16} \mathrm{~s}$.

(a)
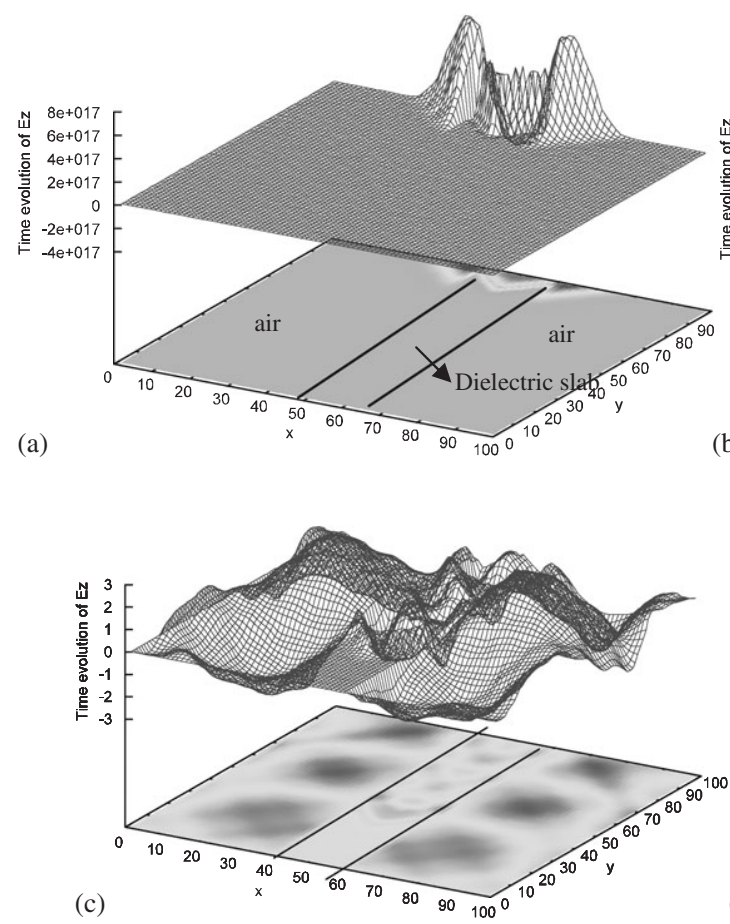
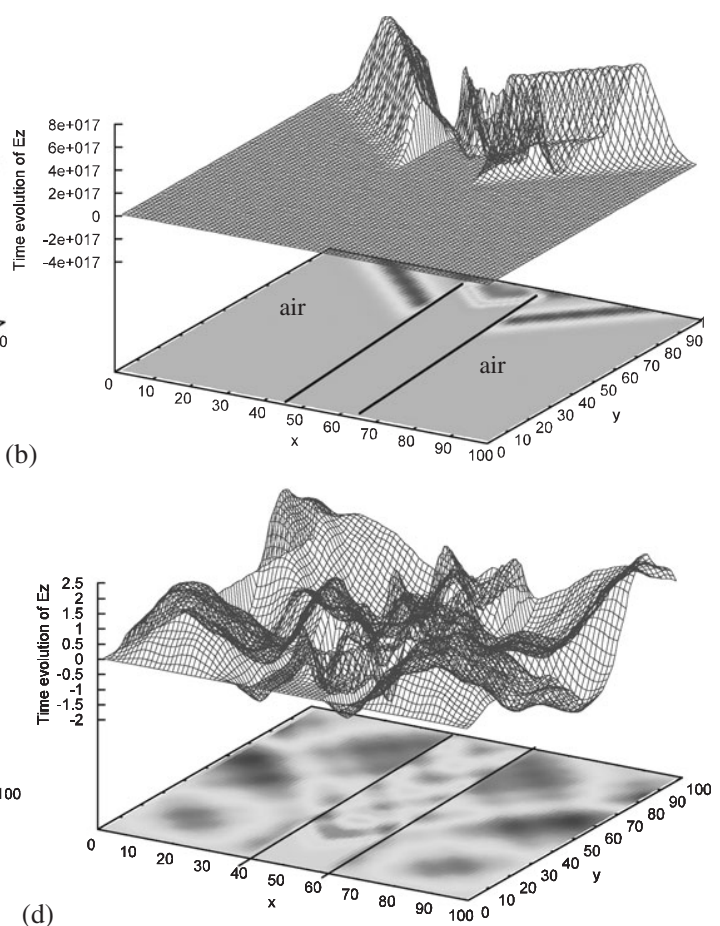

Figure 5. Time evolution of electric $E_{z}$ component in an $\mathrm{Si}(\varepsilon=9)$ slab waveguide in air after (a) 20 timesteps; (b) 50 time-steps; (c) 100 time-steps; and (d) 200 time-steps for a pulsed excitation at $\lambda_{0}=1.55 \mu \mathrm{m}$.

sources used in Equations (24) and (25) are decomposed into two components $\Psi^{\mathrm{e}}$ and $\Psi^{\mathrm{h}}$ [6] that construct the wavefront in the spatial domain. In Figure 5 we show an example of wavefront propagation in a symmetrical slab waveguide: the wavefront is the superposition effect of $\Psi^{\mathrm{e}}$ and $\Psi^{\mathrm{h}}$ sources [6] represented in Equation (25).

\section{TE AND TM ANALYSIS, EFFECTIVE DIELECTRIC CONSTANT (EDC) METHOD, AND FREQUENCY RESPONSE}

The transverse electric (TE) and the transverse magnetic (TM) approach of an optical waveguide are based on the field characterization of these modes. In particular by considering 
the asymmetrical waveguide shown in Figure 1(b) the $E_{y}$ field component characterizes the TE modes and the $E_{x}$ component characterizes the TM modes. The power density [16, 17] along the propagation axis $(z) W(z, t)(\mathrm{W} / \mathrm{m})$ is derived by performing the integration of Poynting's vector

$$
\begin{aligned}
W(z ; t) & =\iint_{\text {cross section }} P(x, y, z ; t) \mathrm{d} x \mathrm{~d} y \\
& =\iint_{\text {cross section }}\left(E_{y} H_{x}+\left(-E_{x} H_{y}\right)\right) \mathrm{d} x \mathrm{~d} y \\
& =W_{\mathrm{TE}}(z ; t)+W_{\mathrm{TM}}(z ; t)
\end{aligned}
$$

By the effective dielectric constant (EDC) method [16] it is possible to analyze complex 3D optical waveguides in a $2 \mathrm{D}$ case [6]. In fact, for example, the ridge waveguide of Figure 1(d) can be analyzed as a 2D waveguide of Figure 1(b) by evaluating the effective refractive index in the $z$-direction. Figure 6 illustrates the EDC method applied to a ridge waveguide and an example of the TE dispersion equation implemented in the HPM solver (the propagation constants are evaluated numerically by the intersection of the $w_{1}$ and $w_{2}$ plots shown in Figure 6(c)). The implemented dispersion equation of the TE modes is $[16,18]$

$$
w_{1}(u)=\frac{\left(u^{2} \tan (2 u)-u \sqrt{v_{1}^{2}-u^{2}}\right)}{u+\tan (2 u) \cdot \sqrt{v_{1}^{2}-u^{2}}}
$$
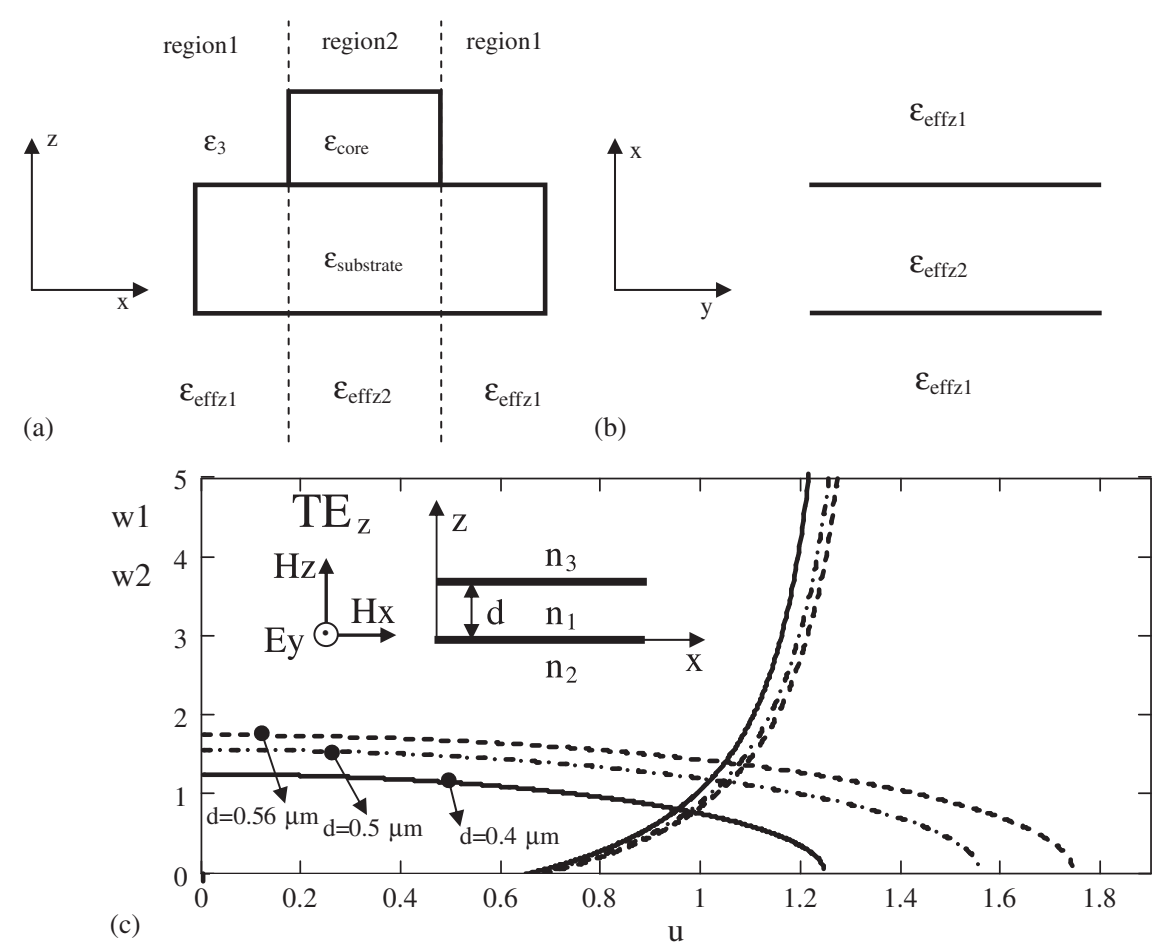

Figure 6. (a) Cross section of ridge waveguide and effective dielectric constants; (b) 2D analysis of the equivalent $3 \mathrm{D}$ structure; and (c) example of $\mathrm{TE}_{\mathrm{z}}$ analysis for different core thicknesses $d$ in an asymmetrical slab waveguide. The working wavelength is $\lambda_{0}=1.55 \mu \mathrm{m}, n_{1}=3.408, n_{2}=3.042, n_{3}$ (air) $=1$. 
where

$$
\begin{aligned}
v_{1} & =k_{0} d \sqrt{\varepsilon_{2}-\varepsilon_{1}} \\
v_{3} & =k_{0} d \sqrt{\varepsilon_{2}-\varepsilon_{3}} \\
w_{2}(u) & =\sqrt{v_{3}^{2}-u^{2}} \\
k_{0} & =\omega \sqrt{\varepsilon_{0} \mu_{0}}
\end{aligned}
$$

and $\varepsilon_{3}=1$ (air superstrate), $\varepsilon_{2}=\varepsilon_{\text {core }}, \varepsilon_{3}=\varepsilon_{\text {substrate }}, k_{0}$ is the wave number in the free space. By using (27) and (28) we evaluate $k_{z}=u / d$ (where $d$ is the core thickness) and then effective index in the $z$-direction $n_{\text {effz }}$ by the wavenumber conservation equation $[18,19]$

$$
k_{0}^{2} \varepsilon_{\text {effz }}=k_{0}^{2} \varepsilon_{2}-k_{z}^{2}
$$

The transverse propagation constant $k_{x}$ instead is obtained by considering the dispersion equations of the symmetrical waveguide $[14,19]$ in the $x$-direction (Figure $6(\mathrm{~b})$ ), and $\varepsilon_{\text {effz }}=n_{\text {effz }}^{2}$ of Equation (29) as effective indices.

In order to define the frequency response, we consider the discrete Fourier transform (DFT). The scattering parameters $S_{m, n}$ can be obtained as follows [14]:

$$
S_{m, n}\left(\omega, y_{m}, y_{n}\right)=\frac{\hat{E}_{m}\left(\omega, y_{m}\right)}{\hat{E}_{n}\left(\omega, y_{n}\right)} \sqrt{\frac{Z_{0, n}(\omega)}{Z_{0, n}(\omega)}}
$$

where $\hat{E}_{m}$ is the phasor voltage (DFT of $E_{z}$ component) at the port $m$ at observation plane $y_{m} ; \hat{E}_{n}$ is the phasor voltage at the port $n$ at observation plane $y_{n}$; and $Z_{0, m}$ and $Z_{0, n}$ are the characteristic impedances of the transmission lines connected to these ports defined as:

$$
Z_{0}\left(\omega, y_{i}\right)=\operatorname{DFT}\left(E_{z}, y_{i}\right) / \operatorname{DFT}\left(H_{x}, y_{i}\right)
$$

In Figure 7 we show an example of the $S_{21}$ transmission parameter related to the optical waveguide of Figure 1(c) for different $\alpha$ angles. We validate the model presented in this work (analytical model by using effective index and numerical generators model) by comparing the Central Processing Unit time for different numerical methods. The computation time comparison is reported in Table I for different tapered waveguides with $L_{1}=L_{2}=5 \mu \mathrm{m}$ (Figure 1(c)), and for a 1-GHz PC, 512/MRAM. In this comparison, we consider the 2D FDTD method (with effective index approach and $\Delta x=\Delta y=0.01 \mu \mathrm{m}$ ), and the 3D FEM method. In

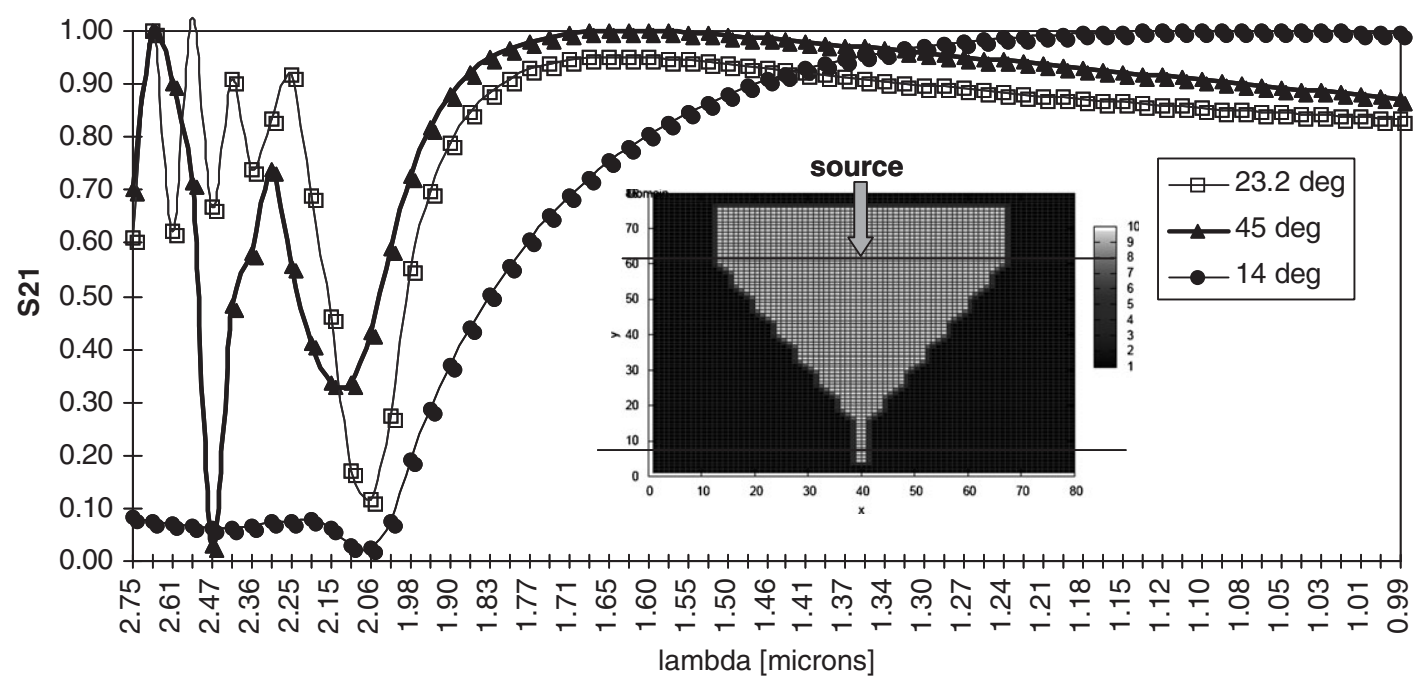

Figure 7. $S_{21}$ scattering parameter for a tapered waveguide $(n=3.067)$ with thickness of the input slab of $5.94 \mu \mathrm{m}$, thickness of the output slab of $0.22 \mu \mathrm{m}, \alpha=14^{\circ}, \alpha=45^{\circ}, \alpha=23.2^{\circ}$. The cell size is $\Delta x=\Delta y=0.11 \mu \mathrm{m}, \mathrm{d} t=3.67 \times 10^{-16} \mathrm{~s}$. 
order to compare the 2D Hertzian Potentials solution with the 3D FEM one, we refine the length of tetrahedral elements (FEM mesh) below the specified value of $0.01 \mu \mathrm{m}$.

\section{DISSIPATIVE MEDIUM AND QUALITY FACTOR $Q$}

The material losses in the metallic and in the dielectric structures are characterized by Equation (8) in which the electric conductivity may represent an ideal dielectric material $(\sigma=0)$ or a perfect metallic material $(\sigma=\infty)$. In order to observe the spatial dielectric loss, we excite with a Gaussian pulse signal defined as:

$$
\Psi_{\text {source }}^{\mathrm{e}, \mathrm{h}}=\exp \left(-\left(t \cdot \mathrm{d} t / T_{0}\right)^{2}\right)
$$

In Figure 8 we show the time evolution of a Gaussian pulse signal in different positions after some cells far from the source. The signal propagates in the dielectric material with an amplitude which decreases with the distance. In Figure 9 the spatial configuration of the exponential signal after different time steps is illustrated. It is evident by analyzing the evolution of the signal for different positions how the signal is attenuated. We use in the $1 \mathrm{D}$ simulation $\Delta z=10^{-8} \mathrm{~m}, \mathrm{~d} t=6.67 \times 10^{-17} \mathrm{~s}, T_{0}=0.1 \times 10^{-17} \mathrm{~s}$ and the source (32) is centered in the dielectric material.

The losses of a non-dissipative dielectric periodic struture such as dielectric multilayer stack and cavity resonators are evaluated by the introduction of the quality factor $Q$. The general expression of the $Q$ factor of any resonator is given by [17]

$$
Q=\frac{\omega_{0} \cdot(\text { Energy stored })}{(\text { Average power loss) }}=\frac{\omega_{0} \cdot U}{W_{L}}
$$

where $U$ is computed inside the volume $V$ of the cavity as:

$$
U=\frac{\varepsilon}{2} \cdot \int_{V}|\mathbf{E}|^{2} \mathrm{~d} V
$$

Table I. Comparison between CPU time for the 2D Hertzian potentials method (HPM), FDTD 2D, and 3D FEM method regarding the results of Figure 7.

\begin{tabular}{lcccc}
\hline$\alpha($ deg.) & $\mu(\mathrm{mm})$ & CPU time 2D HPM (s) & CPU time 2D FDTD (s) & CPU time 3D FEM (min) \\
\hline 14 & 16.22 & 1.9 & 4.3 & 42 \\
23.2 & 6.73 & 1.3 & 2.9 & 38 \\
45 & 2.86 & 0.7 & 1.6 & 25 \\
\hline
\end{tabular}

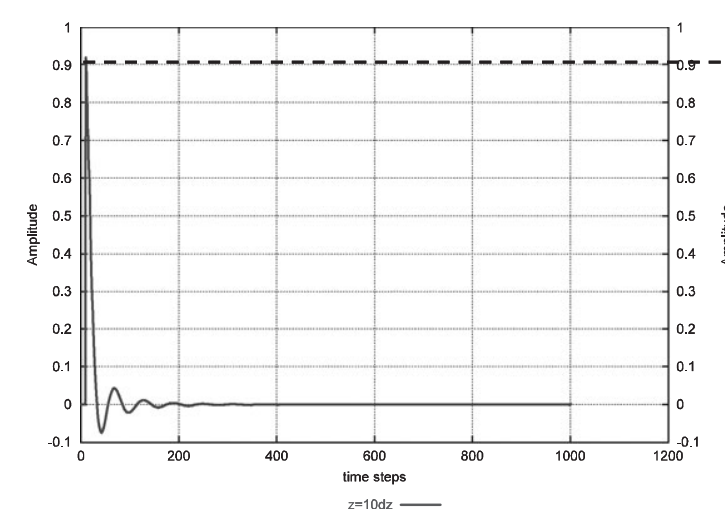

Figure 8. 1D simulation: time

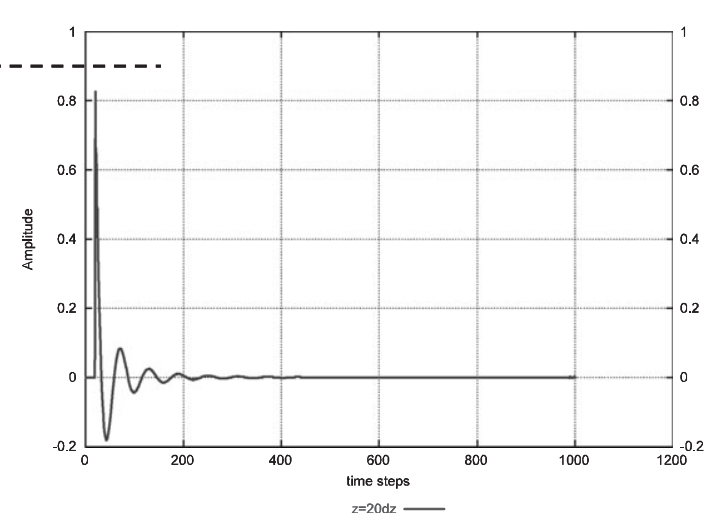

evolution of a Gaussian pulse signal after 10 (left) and 20 (right) cells from the source. 


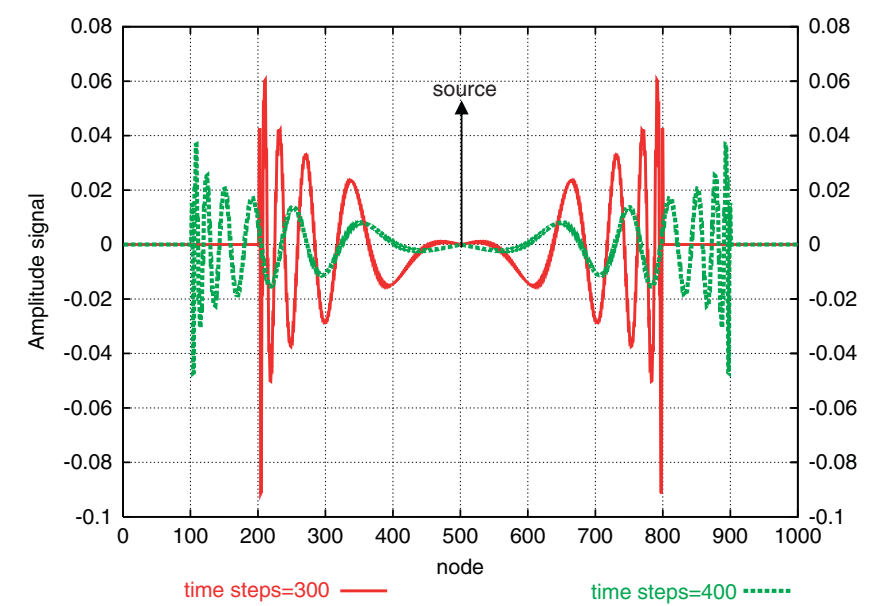

Figure 9. Spatial configuration $\left(n_{\mathrm{z}}=1000\right)$ for 300 and 400 time steps in a material characterized by $\varepsilon=4$, $\sigma=1.061 \times 10^{-6} \mathrm{~S} / \mathrm{m}$.

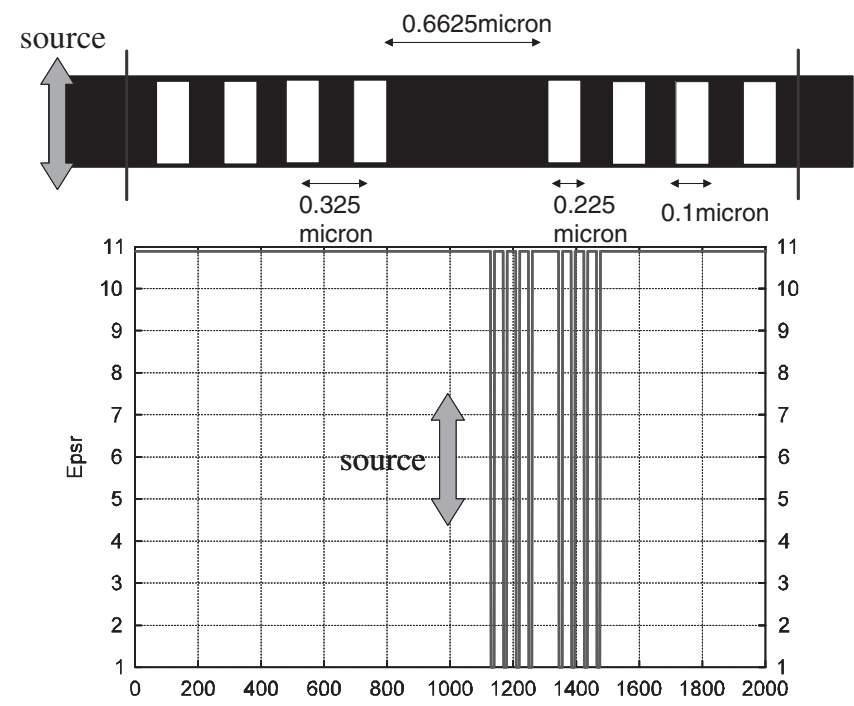

Figure 10. Relative dielectric permittivity in a quasi one-dimensional photonic band gap structure (rectangular air holes in semiconductor waveguide with refractive index $n=3.3$ ).

By supposing a $y$-propagation direction it is possible to evaluate $W_{L}$ (dissipated power) by Poynting's vector

$$
P_{y}(x, y, z ; t)=E_{z} H_{x}-E_{x} H_{z}
$$

By evaluating (35) in the case of an homogeneous structure (uniform guide without discontinuities) and in the case of an inhomogeneous structure (guide with discontinuities), it is possible to evaluate $W_{L}$ as the difference of the two cases.

In Figure 10 an example of a quasi one-dimensional photonic band gap structure [14] is reported. As expected the maximum $\mathrm{Q}$ factor is obtained near the resonance of the cavity mode at $\lambda=1.595 \mu \mathrm{m}$ (Figure 11).

\section{IMPLEMENTATION OF BERENGER'S PERFECTLY MATCHED LAYER}

The HPM simulator employs Berenger's perfectly matched layer (PML) [20] to absorb radiated waves and guided modes at domain boundaries. The continuous time-domain differential 


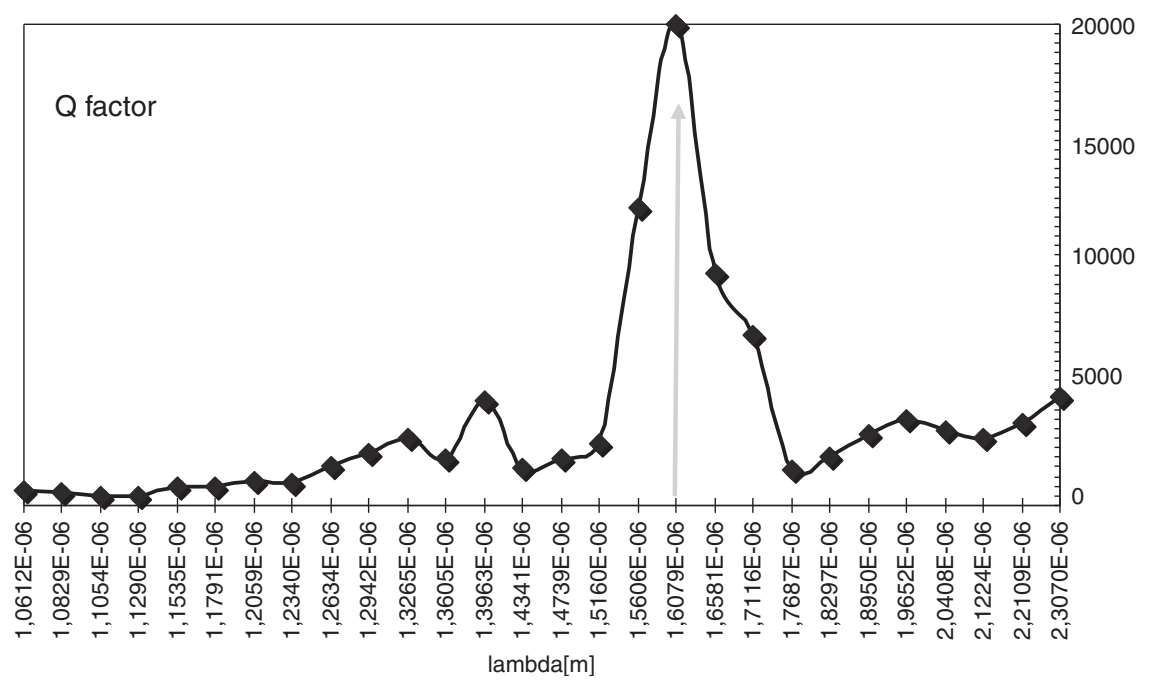

Figure 11. Quality $Q$ factor of the quasi one-dimensional photonic band gap structure.

equations governing fields in the PML are

$$
\begin{array}{ll}
\frac{\mu_{r}}{c_{0}} \eta_{0} \frac{\partial H_{x z}}{\partial t}+\frac{\sigma_{z}^{*}}{\eta_{0}} \eta_{0} H_{x z}=\frac{\partial E_{y}}{\partial z}, & \frac{\varepsilon_{r}}{c_{0}} \frac{\partial E_{x y}}{\partial t}+\sigma_{y} \eta_{0} E_{x y}=\eta_{0} \frac{\partial H_{z}}{\partial y} \\
\frac{\mu_{r}}{c_{0}} \eta_{0} \frac{\partial H_{x y}}{\partial t}+\frac{\sigma_{y}^{*}}{\eta_{0}} \eta_{0} H_{x y}=-\frac{\partial E_{z}}{\partial y}, & \frac{\varepsilon_{r}}{c_{0}} \frac{\partial E_{x z}}{\partial t}+\sigma_{z} \eta_{0} E_{x z}=-\eta_{0} \frac{\partial H_{y}}{\partial z} \\
\frac{\mu_{r}}{c_{0}} \eta_{0} \frac{\partial H_{y x}}{\partial t}+\frac{\sigma_{x}^{*}}{\eta_{0}} \eta_{0} H_{y x}=\frac{\partial E_{z}}{\partial x}, & \frac{\varepsilon_{r}}{c_{0}} \frac{\partial E_{y z}}{\partial t}+\sigma_{z} \eta_{0} E_{y z}=\eta_{0} \frac{\partial H_{x}}{\partial z} \\
\frac{\mu_{r}}{c_{0}} \eta_{0} \frac{\partial H_{y z}}{\partial t}+\frac{\sigma_{z}^{*}}{\eta_{0}} \eta_{0} H_{y z}=-\frac{\partial E_{x}}{\partial z}, & \frac{\varepsilon_{r}}{c_{0}} \frac{\partial E_{y x}}{\partial t}+\sigma_{x} \eta_{0} E_{y x}=-\eta_{0} \frac{\partial H_{z}}{\partial x} \\
\frac{\mu_{r}}{c_{0}} \eta_{0} \frac{\partial H_{z y}}{\partial t}+\frac{\sigma_{y}^{*}}{\eta_{0}} \eta_{0} H_{z y}=\frac{\partial E_{x}}{\partial y}, & \frac{\varepsilon_{r}}{c_{0}} \frac{\partial E_{z x}}{\partial t}+\sigma_{x} \eta_{0} E_{z x}=\eta_{0} \frac{\partial H_{y}}{\partial x} \\
\frac{\mu_{r}}{c_{0}} \eta_{0} \frac{\partial H_{z x}}{\partial t}+\frac{\sigma_{x}^{*}}{\eta_{0}} \eta_{0} H_{z x}=-\frac{\partial E_{y}}{\partial x}, & \frac{\varepsilon_{r}}{c_{0}} \frac{\partial E_{z y}}{\partial t}+\sigma_{y} \eta_{0} E_{z y}=-\eta_{0} \frac{\partial H_{x}}{\partial y}
\end{array}
$$

Assuming $\exp (\mathrm{j} \omega t)$ time variation, and combining split components result in

$$
\begin{array}{rlrl}
H_{x} & =a_{z} \frac{\partial E_{y}}{\partial z}-a_{y} \frac{\partial E_{z}}{\partial y}, & E_{x}=b_{y} \frac{\partial H_{z}}{\partial y}-b_{z} \frac{\partial H_{y}}{\partial z} \\
H_{y}=a_{x} \frac{\partial E_{z}}{\partial x}-a_{z} \frac{\partial E_{x}}{\partial z}, & E_{y}=b_{z} \frac{\partial H_{x}}{\partial z}-b_{x} \frac{\partial H_{z}}{\partial x} \\
H_{z}=a_{y} \frac{\partial E_{x}}{\partial y}-a_{x} \frac{\partial E_{y}}{\partial x}, & E_{z}=b_{x} \frac{\partial H_{y}}{\partial x}-b_{y} \frac{\partial H_{x}}{\partial y}
\end{array}
$$

where

$$
\begin{aligned}
a_{i} & =\frac{1}{\mathrm{j} \omega \mu_{r} / c_{0}+\eta_{0} \sigma_{i}^{*}} \\
b_{i} & =\frac{1}{\mathrm{j} \omega \varepsilon_{r} / c_{0}+\eta_{0} \sigma_{i}}
\end{aligned}
$$

and the EM field components are provided by Equation (6). Figure 12 depicts the placement strategy of the PML. The code in this work used an $n$ th-order conductivity 


\begin{tabular}{|c|c|c|}
\hline $\begin{array}{c}\sigma_{x} \\
\sigma_{y} \\
\varepsilon_{2}, \mu_{2}\end{array}$ & 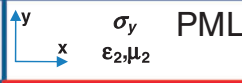 & $\begin{array}{c}\sigma_{x} \\
\sigma_{y} \\
\varepsilon_{2}, \mu_{2}\end{array}$ \\
\hline $\begin{array}{c}\sigma_{x} \\
\varepsilon_{2,}, \mu_{2}\end{array}$ & $\varepsilon_{2}, \mu_{2}$ & $\begin{array}{c}\sigma_{x} \\
\varepsilon_{2}, \mu_{2}\end{array}$ \\
\hline $\begin{array}{c}\sigma_{x} \\
\varepsilon_{1}, \mu_{1}\end{array}$ & $\left.\varepsilon_{1}, \mu_{1}\right\rceil^{\text {source }}$ & $\begin{array}{c}\sigma_{x} \\
\varepsilon_{1}, \mu_{1}\end{array}$ \\
\hline $\begin{array}{c}\sigma_{x} \\
\varepsilon_{2}, \mu_{2}\end{array}$ & $\varepsilon_{2}, \mu_{2}$ & $\begin{array}{c}\sigma_{x} \\
\varepsilon_{2}, \mu_{2}\end{array}$ \\
\hline $\begin{array}{c}\sigma_{x} \\
\sigma_{y} \\
\varepsilon_{2}, \mu_{2}\end{array}$ & $\begin{array}{c}\sigma_{y} \\
\varepsilon_{2}, \mu_{2}\end{array}$ & $\begin{array}{c}\sigma_{x} \\
\sigma_{y} \\
\varepsilon_{2}, \mu_{2}\end{array}$ \\
\hline
\end{tabular}

(a)

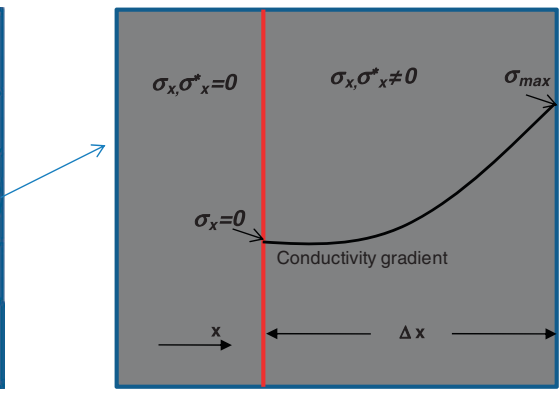

(b)

Figure 12. The PMLs are placed as depicted in (a) with the $\mathbf{z}$ PMLs following the same convention. The conductivity gradient in the PML is depicted in (b) with the associated parameters.

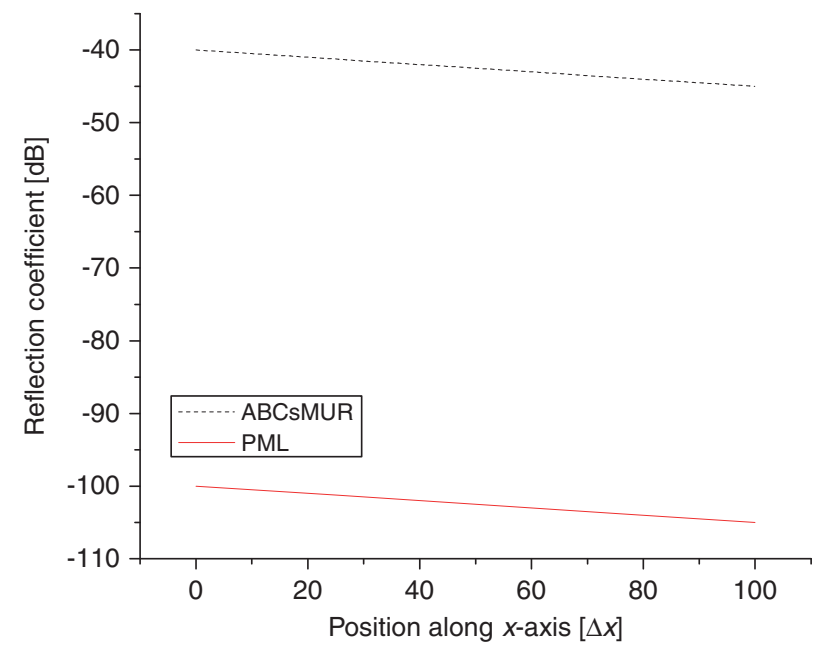

Figure 13. Reflection coefficients of the absorbing boundaries: Berenger's PML and ABCs Mur. The simulation is performed by considering $\Delta x=\Delta y=0.01 \mu \mathrm{m}$ with $\sigma_{i}$ varying quadratically in the $x$-, and $y$-direction.

gradient defined as

$$
\sigma_{i}(i)=\sigma_{\max }\left(\frac{i}{\Delta i}\right)^{n}, \quad i=x, y, z
$$

where $\Delta i$ is the width of the PML.

In Figure 13 we show the comparison between the reflection coefficients of the ABCs Mur and that of the PML: the simulation concerns the propagation of the modulated pulse of Figure 4 propagating in the Si $\left(\varepsilon_{1}=9\right)$ slab waveguide of Figure 12(a). In this analysis, PML has a very small reflection coefficient of $-105 \mathrm{~dB}$ if compared with the results of the ABCs Mur approach.

\section{CONCLUSION}

The HPM method combines the analytical approach for optical integrated waveguides and the numerical advantages of a time-domain with a low computational cost. By using the HPM formulation, this paper presents an accurate investigation of optical waveguides finding wide applications in optics. The HPM method with the effective index method can also be applied for complex and irregular periodic 3D structures by considering the analysis in the $2 \mathrm{D}$ time domain with a low computational cost. The HPM provides an accurate and flexible tool for 
computational optics. Berenger's PML technique is implemented in the proposed tool by optimizing the ABCs.

\section{REFERENCES}

1. Yee KS. Numerical solution of initial boundary value problems involving Maxwell's equation in isotropic media. IEEE Transactions on Antennas Propagation 1966; 14:302-307.

2. Taflove A. Application of the finite-difference time-domain method to sinusoidal steadystate electromagnetic penetration problems. IEEE Transactions on Electromagnetic Compatibility 1980; 22:191-202.

3. Hoefer WJR. The transmissionline matrix method - theory and applications. IEEE Transactions on Microwave Theory and Techniques 1985; 33:882-893.

4. Fujii M, Hoefer WJR. A threedimensional Haar-wavelet-based multiresolution analysis similar to the FDTD method - derivation and application. IEEE Transactions on Microwave Theory and Techniques 1998; 46(12): 2463-2475.

5. Massaro A, Rozzi T. Rigorous time-domain analysis of dielectric optical waveguides using Hertzian potentials formulation. Optics Express 2006; 14(5): 2027-2036.

6. Massaro A, Grande M, Cingolani $\mathrm{R}$, Passaseo A, De Vittorio M. Design and modelling of tapered waveguide for photonic crystal slab coupling by using timedomain Hertzian potential formulation. Optics Express. 2007; 15(25):16484-16499.

7. Couture M. On the numerical solution of fields in cavities using the magnetic Hertz vector. IEEE Transactions on Microwave Theory and Techniques 1987; 35(3):288-295.

8. Nikoskinen KI. Time-domain study of arbitrary dipole in planar geometry with discontinuity in permittivity and permeability. IEEE Transactions on Antennas Propagation 1991; 39(5):698-703.

9. Rozzi T, Farina M. Advanced Electromagnetic Analysis of Passive and Active Planar Structures. IEE Electromagnetic Wave Series, vol. 46. IEE: London, 1999.

10. Someda CG. Onde Elettromagnetiche. UTET: Torino, 1996.

11. Marcuvitz N, Schwinger J. On the representation of the electric and magnetic field produced by currents and discontinuities in wave guides. Journal of Applied Physics 1951; 22(6): 806-820.

12. Frateschi NC, Rubens A, De Castro B. Perturbation theory for the wave equation and the effective refractive index approach. IEEE Journal of Quantum Electronics 1986; 22(1): $12-15$.

13. Yariv A. Quantum Electronics (3rd edn). Wiley: Canada, 1989.

14. Taflove A, Hagness SC. Computational Electrodynamic: The
Finite-difference Time-domain Method (2nd edn). Arthec House Publishers: London, 2000.

15. Taflove A, Brodwin ME. Numerical solution of steady-state electromagnetic scattering problems using the time-dependent Maxwell's equations. IEEE Transactions on Microwave Theory and Techniques 1975; 23:623-630.

16. Rozzi T, Mongiardo M. Open Electromagnetic Waveguides. IEE Electromagnetic Wave Series, vol. 43. IEE: London, 1997.

17. Pierantoni L, Di Donato A, Rozzi T. Full-wave analysis of photonic bandgap integrated optical components by the TLM-IE method. IEEE Journal of Lightwave Technology 2004; 22(10):2348-2358.

18. Marcuse D. Theory of Dielectric Opt. Waveguides. Academic Press: New York, 1974.

19. Mur G. Absorbing boundary conditions for the finitedifference approximation of the time-domain electromagnetic field equations. IEEE Transactions on Electromagnetic Compatibility 1981; 23:377-382.

20. Berenger J-P. A perfectly matched layer for the absorption of electromagnetic waves. Journal of Computational Physics 1994; 114:185-200.

\section{AUTHORS' BIOGRAPHIES}

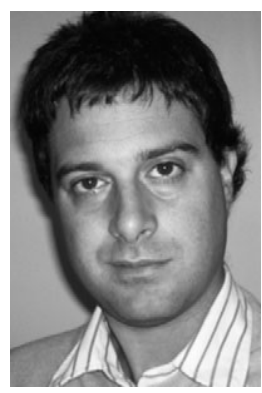

Alessandro Massaro received the Laurea degree in Electronic Engineering and the $\mathrm{PhD}$ degree in Telecommunication Engineering from the Università Politecnica delle Marche, Ancona, Italy, in 2001 and 2004, respectively. From 2004 to 2006 he worked as a Research Scientist (post-doc) in the Department of Electromagnetism and Bioengineering at Università Politecnica delle Marche. In 2006, he spent two years in Research and Development in the medical and industrial optics industry (endoscope design and optical systems). He worked for two years with the National Nanotechnology Laboratory of CNR-INFM as principal investigator. $\mathrm{He}$ is currently the team leader in Robotics Lab. 1 of the Center of Bio-Molecular Nanotechnology of the Italian Institute Technology (IIT), Arnesano, Lecce, Italy. His research interests are in the design and modeling of photonic band gap circuits, in the development of computer aided design (CAD) tools in the area of integrated optics, MEMS/NEMS technology and systems, and smart material design. He is also a member of the European Microwave Association (EuMA). 


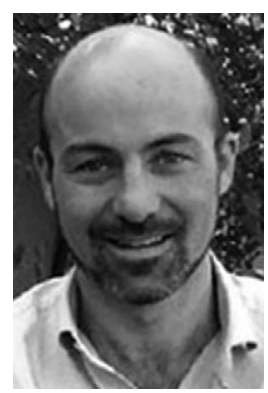

Roberto Cingolani was born in Milan (Italy) in December 1961. He obtained a PhD in Physics at Scuola Normale Superiore di Pisa (Italy) and he was a staff member of Max Planck Institute FKF-Stuttgart from 1989-1991. He was a visiting scientist at the Tokyo University in 1997, and appointed full professor of experimental physics at the University of Salento since 2000. He is the Scientific Director of the Italian Institute of Technology since 2004. He is the author and co-author of more than 500 papers in international journals and he holds 20 patents. His research activity includes nanotechnology and nanofabrication, semiconductor physics and devices, molecular organic nanostructures and devices, and bio-nanotechnologies. 